\title{
A novel and remote biochemical verification method of smoking abstinence: Predictors of participant compliance
}

\author{
Johannes Thrul', Meredith C. Meacham², Danielle E. Ramo²
}

\begin{abstract}
INTRODUCTION Biochemical verification of smoking abstinence remains an important validity check of cessation trial outcomes. Digital health trials rarely establish inperson contacts between participants and intervention providers, requiring novel strategies to biochemically verify outcomes. We describe remote verification of smoking abstinence via saliva cotinine and individual predictors of compliance in a digital intervention.

METHODS Data came from a feasibility trial and randomized controlled trial of a Facebook smoking cessation intervention for young adults. In both trials, participants completed baseline and follow-up surveys at 3, 6 and 12 months. Participants indicating past 7 -day point prevalence smoking abstinence were mailed a saliva cotinine kit. Participants were instructed to electronically send two photos - one of them giving a saliva sample and the other with the test results. We investigated predictors of compliance with these procedures, independent of verification results, among participants that were mailed a kit at any follow-up point $(\mathrm{N}=130$; mean age $=21.3 ; 59.2 \%$ female $)$ using logistic and multinomial regression. RESULTS A total of 189 kits were sent out, of which 97 were completed $(51.3 \%$ compliance). We did not identify significant predictors of completing any vs no kits using logistic regression. We also found no significant predictors of extent of kit completion (none vs some; none vs all) using multinomial regression and controlling for number of kits sent.

CONCLUSIONS Findings demonstrate the feasibility of this biochemical verification method and suggest low risk for bias of results. Future studies should replicate findings in larger samples and improve compliance with verification procedures.
\end{abstract}

AFFILIATION

1 Department of Mental Health, Johns Hopkins Bloomberg School of Public Health, Baltimore, USA 2 Department of Psychiatry and Weill Institute for Neurosciences, University of California San Francisco, San Francisco, USA

CORRESPONDENCE TO Johannes Thrul. Johns Hopkins Bloomberg School of Public Health, 624 N Broadway, 21205 Baltimore, United States. E-mail: jthrul@jhu.edu

\section{KEYWORDS}

validation, young adults, social media, cessation

Received: 12 February 2018 Revised: 6 April 2018

Accepted: 30 April 2018

\section{INTRODUCTION}

Biochemical verification of smoking abstinence remains an important validity check of smoking cessation trial outcomes ${ }^{1-3}$. Reliance on selfreported smoking status may lead to over-reporting of smoking abstinence due to inclinations to report socially desirable behavior, especially in the context of intervention trials ${ }^{2}$. Salivary cotinine is commonly used $^{4}$ while other methods include more expensive expiratory carbon monoxide ( $\mathrm{CO}$ ) testing and more invasive urinary or serum cotinine testing. Biochemical verification can be costly and time intensive, and a burden to participants who may be required to travel to a field site or clinic to provide the required bio-sample.

Biochemical verification of intervention outcomes is especially challenging in the context of digital smoking cessation interventions. Interventions delivered via text message, smartphone apps or social media have the potential to provide smoking cessation treatment and support to a large number of smokers from geographically diverse locations $\mathrm{s}^{3,5}$, yet they rarely establish in-person contact between participants and intervention providers. Therefore, 
strategies are needed to implement biochemical verification of abstinence remotely ${ }^{6}$.

Biochemical verification methods in digital smoking cessation trials have made use of both salivary cotinine and carbon monoxide breath samples, yet remote testing is rare. One text-message based intervention required in-person assessment of biochemically verified abstinence ${ }^{7}$, while others used postal salivary cotinine testing in which participants were mailed kits and had to return a saliva sample by mail ${ }^{8,9}$. A few studies reported using videos to verify use of a $\mathrm{CO}$ monitor and online reporting of $\mathrm{CO}$ value ${ }^{10,11}$. However, the need to send costly equipment through the mail limits the study sample size.

Few digital smoking cessation trials have reported participant compliance with biochemical verification procedures. Studies of text message cessation trials in the general population report rates of biochemical verification compliance through mail that range from $64.7 \%^{8}$ to $81.3 \%{ }^{9}$, and do not report participant level predictors of compliance. An even lower proportion of participants (39.2\%) who self-reported abstinence attended an in-person salivary cotinine testing verification visit as part of a mobile phone text messaging intervention trial ${ }^{7}$. Only one study has reported participant level predictors of compliance. In a study of Veterans Administration patients receiving an in-patient smoking cessation intervention, the $90 \%$ of patients who returned a urinary cotinine strip by mail at the 6-month follow up were younger, more likely to be thinking about quitting, more likely to have arthritis, and less likely to have heart disease compared to patients who did not return a test strip ${ }^{12}$. In the absence of predictors of biochemical verification compliance for digital smoking cessation trials, it is unclear how representative obtained results are.

We describe here a novel method for verifying smoking abstinence through remote testing of salivary cotinine and verification via digital photos, among young adults participating in a Facebook smoking cessation intervention. We also investigated whether baseline demographics, smoking behavior and readiness to quit smoking might explain differences in compliance with biochemical verification procedures.

\section{METHODS}

\section{Procedure}

Data came from two trials investigating the feasibility and efficacy of the Tobacco Status Project (TSP) - a motivationally-tailored smoking cessation intervention delivered through Facebook. First, a feasibility trial $(\mathrm{N}=79)^{13-15}$ assigned participants to a TSP intervention group. In the second study, a Randomized Controlled Trial (RCT, N=500) ${ }^{16,17}$, participants were randomized to one of two conditions: 1) the TSP intervention, or 2) a control condition (referral to the Smokefree.gov website). TSP was delivered entirely on Facebook and consisted of motivationally-tailored automated daily posts to secret Facebook groups and weekly live smoking cessation counselor contact. In both studies, assessments were conducted online at baseline and at 3, 6 and 12 months follow-up. Participants received their choice of gift cards of $\$ 20$ per survey assessment, and a $\$ 20$ bonus for completing all three assessments, for a possible total incentive of $\$ 100$. At every follow-up assessment (at 3,6 and 12 months) participants who reported past 7-day point prevalence abstinence were mailed a NicAlert saliva cotinine test kit (Confirm Biosciences) with study-designed instructions for its use (see Appendix 1), with a maximum of 3 test kits per participant. Participants were instructed to take two photos: one of the participant giving the saliva sample and the other of the strip showing the resulting cotinine range (from Level 0, corresponding to the range $0-10 \mathrm{ng} / \mathrm{mL}$, to Level 6 , in the range $1000+\mathrm{ng} / \mathrm{mL}$ ). The two photos were then emailed or sent by Facebook messenger to study coordinators. If biochemical verification was requested, the $\$ 20$ gift card was only sent if participants also completed verification procedures in addition to the survey. There was no incentive given for reporting abstinence. All study procedures were approved by the Institutional Review Board of the University of California San Francisco.

\section{Participants}

Participants were literate in English, aged 18-25 years, reported smoking $\geqslant 100$ cigarettes in their lifetime and smoking at least 1 cigarette per day on 3 or more days of the week. Intention to quit smoking was not required for trial enrollment. Additional inclusion criteria were regular ( $\geqslant 4$ days/week) 
Facebook use and access to a digital camera to verify smoking abstinence. For the current analyses we included only participants that ever self-reported smoking abstinence during any of the follow-up assessments and were sent a biochemical verification kit $(\mathrm{N}=130 ; \mathrm{n}=21$ feasibility; $\mathrm{n}=109$ RCT $)$.

\section{Measures}

\section{Baseline}

Measures included age, gender, race/ethnicity, completed education, annual income, employment and Smoking History Questionnaire ${ }^{18}$, including time of first cigarette on waking up ( $\leqslant 30 \mathrm{~min}$ or $<30 \mathrm{~min}$ ) to assess nicotine dependence ${ }^{19}$ and a measure of smoking cessation goals ${ }^{20}$.

\section{Biochemical verification}

The primary outcome was compliance with biochemical verification procedures at 3, 6 and 12 months, defined as study receipt of two digital photos, clearly showing the participant giving the saliva sample (Appendix photo 1) and the resulting cotinine range (Appendix photo 2), regardless of the cotinine result.

\section{Statistical analyses}

First, predictors of compliance with biochemical verification procedures at any follow-up point (any vs none) were modelled using logistic regression. Second, extent of biochemical verification compliance (none, some, all) over 12 months was modelled using multinomial regressions with 'none' as the reference category. To account for the fact that participants who more frequently self-reported abstinence were mailed more kits, these analyses additionally controlled for number of kits that were mailed to a given participant. There was no difference in compliance by condition $\left(\chi^{2}[1]=0.05, \mathrm{p}=0.83\right)$; thus condition was not included in the regression analyses.

\section{RESULTS}

Participant characteristics are reported in Table 1. A total of 189 biochemical verification kits were sent out to participants throughout the follow-up period of each trial (Feasibility: 33; RCT: 156), of which 97 (51.3\%) were completed (Feasibility: 17; $51.5 \%$; RCT: $80 ; 51.2 \%)$ with $85(87.7 \%)$ of these
Table 1: Sample description and logistic regression results predicting any compliance with biochemical verification procedures ${ }^{l}(\mathrm{~N}=130)$

\begin{tabular}{|c|c|c|c|c|}
\hline & $\begin{array}{l}\text { Nor } \\
\text { mean }\end{array}$ & $\begin{array}{l}\text { oor } \\
\text { SD }\end{array}$ & OR & $95 \% \mathrm{CI}$ \\
\hline Age $(M, S D)$ & 21.3 & 2.2 & 1.0 & $0.9-1.2$ \\
\hline Gender identity: female & 77 & $59.2 \%$ & 0.5 & $0.3-1.1$ \\
\hline Race/Ethnicity Non-White & 35 & $26.9 \%$ & 1.0 & $0.5-2.3$ \\
\hline \multicolumn{5}{|l|}{ Household Income } \\
\hline$\$ 20000$ or less & 38 & $29.2 \%$ & Ref. & \\
\hline$\$ 21000-40000$ & 41 & $31.5 \%$ & 0.8 & $0.3-1.8$ \\
\hline$\$ 41000-60000$ & 18 & $13.8 \%$ & 0.4 & $0.1-1.3$ \\
\hline$\$ 61000$ or more & 33 & $25.4 \%$ & 0.8 & $0.3-2.0$ \\
\hline \multicolumn{5}{|l|}{ Highest level of education } \\
\hline Less than high school & 12 & $9.2 \%$ & Ref. & \\
\hline Complete high school/GED & 30 & $23.1 \%$ & 0.4 & $0.1-1.8$ \\
\hline Some college & 81 & $62.3 \%$ & 0.6 & $0.2-2.2$ \\
\hline Completed college & 7 & $5.4 \%$ & 0.4 & $0.1-2.6$ \\
\hline \multicolumn{5}{|l|}{$\begin{array}{l}\text { Employment status } \\
\text { (compared to employed } \\
\text { full-time) }\end{array}$} \\
\hline Employed full-time & 54 & $41.5 \%$ & Ref. & \\
\hline Employed part-time & 31 & $23.9 \%$ & 0.7 & $0.2-4.2$ \\
\hline Unemployed & 45 & $34.6 \%$ & 1.0 & $0.2-3.0$ \\
\hline Daily smoker & 93 & $71.5 \%$ & 0.7 & $0.3-1.6$ \\
\hline $\begin{array}{l}\text { Number of smoking days } \\
\text { per week }(M, S D)\end{array}$ & 6.3 & 1.2 & 1.0 & $0.9-1.0$ \\
\hline $\begin{array}{l}\text { Number of cigarettes per } \\
\text { smoking day }(\mathrm{M}, \mathrm{SD})\end{array}$ & 9.1 & 6.3 & 1.0 & $0.8-1.3$ \\
\hline $\begin{array}{l}\text { Smoke first cigarette } \leq 30 \\
\text { min of waking (TTFC) }\end{array}$ & 57 & $43.9 \%$ & 0.8 & $0.4-1.6$ \\
\hline Any past year quit attempt & 94 & $72.3 \%$ & 1.1 & $0.5-2.3$ \\
\hline \multicolumn{5}{|l|}{ Smoking cessation goal } \\
\hline No goal & 18 & $13.9 \%$ & Ref. & \\
\hline Reduction & 80 & $61.5 \%$ & 1.3 & $0.5-3.6$ \\
\hline Abstinence & 32 & $24.6 \%$ & 1.0 & $0.3-3.2$ \\
\hline \multicolumn{5}{|l|}{ Readiness to quit } \\
\hline Pre-contemplation & 18 & $13.9 \%$ & Ref. & \\
\hline Contemplation & 68 & $52.3 \%$ & 1.3 & $0.4-3.6$ \\
\hline Preparation & 44 & $33.8 \%$ & 2.2 & $0.7-6.7$ \\
\hline
\end{tabular}

$\mathrm{OR}=$ odds ratio; $95 \% \mathrm{Cl}=95 \%$ confidence interval; Ref. = reference category for logistic regression;

1 Biochemical verification of abstinence was considered completed when a participant returned to study staff photos of giving saliva sample and of test results. Biochemical verification was completed by $70 / 130$ participants (53.7\%)

confirmed abstinent (Feasibility: 16/17; 94.1\%; RCT: $69 / 80 ; 86.3 \%)$. Over half of the participants $(n=70$; $53.9 \%$ ) completed a biochemical verification kit at any follow-up. There were no significant participant baseline predictors of any vs no compliance with biochemical verification procedures (Table 1). 
In total, 20 participants (15.4\%) completed some of the kits and 50 participants (38.5\%) completed all of the kits. In multinomial logistic regression analyses, there were no significant participant baseline predictors of extent of compliance with biochemical verification procedures (data not shown).

\section{DISCUSSION}

The current study investigated predictors of compliance with remote biochemical verification of smoking abstinence among young adults participating in two trials of a Facebook smoking cessation intervention. We did not find any significant predictors of biochemical verification compliance or extent of compliance compared to non-compliance among participants who self-reported 7 -day point prevalence smoking abstinence.

We observed an overall compliance rate of $51.3 \%$ with our biochemical verification procedures. This rate is lower than some studies among general population samples of smokers, that required participants to return saliva samples or test strips in the mail. Previous studies with adults motivated to quit smoking and in-person contacts within a system of care (e.g. Veterans Affairs - VA) reported compliance rates of $90 \%{ }^{12}, 81.3 \%{ }^{9}$ and $64.7 \%{ }^{8}$. Our results compare favorably to a study that required participants to attend an in-person session for salivary cotinine testing, which resulted in a compliance rate of $39.2 \%{ }^{7}$. We expected that our method of asking participants to return digital photos of saliva cotinine test results would yield higher compliance than requiring a return through the mail in a young adult population, yet, this was not directly evaluated through our trials. We also did not compare compliance with our saliva test to compliance with urine testing used in other studies ${ }^{12}$ because we anticipated problems with implementing a urine testing procedure remotely in this entirely digital study.

It is unclear how our sample of young adult participants, only some of whom were ready to quit smoking at baseline, may have impacted on observed compliance rates. Previous studies have suggested that it may be more difficult to engage young adults in highly compliant behavior in studies on tobacco use, especially if there is no in-person contact between participants and study staff ${ }^{21}$.

\section{Limitations}

Our sample consisted of young adult smokers participating in a Facebook smoking cessation intervention and findings may not be generalizable to other populations of different ages or levels of experience in using technology. Moreover, we did not use other methods of biochemical verification, including mailing of saliva samples or in-person verification, for direct comparison of compliance rates. Additionally, our analytical sample size of 130 may have limited power to detect significant differences, although 130 is well above the rule of thumb of 10 observations per independent variable in logistic regression analyse ${ }^{22}$, and there was a relatively even distribution between returned (i.e. compliant) and not-returned biochemical verification test kits.

\section{CONCLUSIONS}

Findings are promising and suggest that this method of conducting biochemical verification of smoking cessation intervention outcomes is feasible. There may be low risk of systematic biases regarding how young adult participants comply with verification procedures in the context of a Facebook intervention. Biochemical verification results of a subset of participants can potentially be used to extrapolate to the entire sample of study participants self-reporting smoking abstinence. Future studies are needed to replicate findings in larger samples, directly compare multiple methods of biochemical verification and investigate strategies to improve compliance with verification procedures.

\section{REFERENCES}

1. Connor Gorber S, Schofield-Hurwitz S, Hardt J, et al. The accuracy of self-reported smoking: a systematic review of the relationship between self-reported and cotinine-assessed smoking status. Nicotine Tob Res 2009;11:12-24. doi:10.1093/ntr/ntn010

2. Patrick DL, Cheadle A, Thompson DC, et al. The validity of self-reported smoking: a review and meta-analysis. Am J Public Health 1994;84:1086-93.

3. Whittaker R, McRobbie H, Bullen C, et al. Mobile phone-based interventions for smoking cessation. Cochrane Database Syst Rev 2016;4:CD006611. doi:10.1002/14651858.CD006611.pub4

4. SRNT Subcommittee on Biochemical Verification. Biochemical verification of tobacco use and cessation. Nicotine Tob Res 2002;4:149-59. 


\section{doi:10.1080/14622200210123581}

5. Cha S, Ganz O, Cohn AM, et al. Feasibility of biochemical verification in a web-based smoking cessation study. Addict Behav 2017;73:204-8. doi:10.1016/j.addbeh.2017.05.020

6. McClure EA, Gray KM. The Remote Monitoring of Smoking in Adolescents. Adolesc Psychiatry 2013;3:15662. doi:10.2174/2210676611303020006

7. Rodgers A, Corbett T, Bramley D, et al. Do u smoke after txt? Results of a randomised trial of smoking cessation using mobile phone text messaging. Tob Control 2005;14:255-61. doi:10.1136/tc.2005.011577

8. Abroms LC, Boal AL, Simmens SJ, et al. A randomized trial of Text2Quit: a text messaging program for smoking cessation. Am J Prev Med 2014;47:242-50. doi:10.1016/j.amepre.2014.04.010

9. Free C, Knight R, Robertson S, et al. Smoking cessation support delivered via mobile phone text messaging (txt2stop): a single-blind, randomised trial. Lancet 2011;378:49-55. doi:10.1016/S0140-6736(11)60701-0

10. Dallery J, Raiff BR, Grabinski MJ. Internet-based contingency management to promote smoking cessation: A randomized controlled study. J Appl Behav Anal 2013;46:750-64. doi:10.1002/jaba.89

11. Karelitz JL, Michael VC, Boldry M, et al. Validating Use of Internet-Submitted Carbon Monoxide Values by Video to Determine Quit Status. Nicotine Tob Res 2017;19:990-3. doi:10.1093/ntr/ntw311

12. Noonan D, Jiang Y, Duffy SA. Utility of biochemical verification of tobacco cessation in the Department of Veterans Affairs. Addict Behav 2013;38:1792-5. doi:10.1016/j.addbeh.2012.11.006

13 Ramo DE, Thrul J, Chavez K, et al. Feasibility and Quit Rates of the Tobacco Status Project: A Facebook Smoking Cessation Intervention for Young Adults. J Med Internet Res 2015;17:e291. doi:10.2196/jmir.5209

14 Thrul J, Klein AB, Ramo DE. Smoking Cessation Intervention on Facebook: Which Content Generates the Best Engagement? J Med Internet Res 2015;17:e244. doi:10.2196/jmir.4575

15 Thrul J, Ramo DE. Cessation Strategies Young Adult Smokers Use After Participating in a Facebook Intervention. Subst Use Misuse 2017;52:259-64. doi:10.1080/10826084.2016.1223690

16 Ramo DE, Thrul J, Delucchi KL, et al. The Tobacco Status Project (TSP): Study protocol for a randomized controlled trial of a Facebook smoking cessation intervention for young adults. BMC Public Health 2015;15:897. doi:10.1186/s12889-015-2217-0

17 Ramo DE, Thrul J, Delucchi KL, et al. A Randomized Controlled Evaluation of the Tobacco Status Project, a Facebook Intervention for Young Adults. Addiction In press.

18. Hall SM, Tsoh JY, Prochaska JJ, et al. Treatment for Cigarette Smoking Among Depressed Mental Health Outpatients: A Randomized Clinical
Trial. Am J Public Health 2006;96:1808-14. doi:10.2105/AJPH.2005.080382

19. Baker TB, Piper ME, McCarthy DE, et al. Time to First Cigarette in the Morning as an Index of Ability to Quit Smoking: Implications for Nicotine Dependence. Nicotine Tob Res 2007;9:S555-70. doi:10.1080/14622200701673480

20. Thrul J, Stemmler M, Bühler A, et al. The role of participants' self-selected future smoking goals in adolescent smoking cessation interventions. Drug Alcohol Depend 2014;141:118-23. doi:10.1016/j.drugalcdep.2014.05.016

21. Thrul J, Bühler A, Ferguson SG. An Internet-based ecological momentary assessment study relying on participants' own mobile phones: insights from a study with young adult smokers. Eur Addict Res 2015;21:1-5. doi:10.1159/000363231

22. Agresti A. An introduction to categorical data analysis. 2nd ed. Hoboken, NJ: : John Wiley \& Sons, Inc. 2007.
CONFLICTS OF INTEREST D.E. Ramo reports grants from the National Institute on Drug Abuse, and also during the conduct of the study she has consulted for Carrot Inc., which makes a tobacco cessation device. J. Thrul reports grants from the California TobaccoRelated Disease Research Program (TRDRP) during the conduct of the study. M.C. Meacham, also has completed and submitted an ICMJE form for disclosure of potential conflicts of interest. The authors declare that they have no competing interests, financial or otherwise, related to the current work.

\section{FUNDING}

This work was supported by the National Institutes of Health, National Institute on Drug Abuse (NIDA, K23 DA032578). Preparation of this manuscript was, in part, supported by NIDA (T32 DA007250) and the California Tobacco-Related Disease Research Program (TRDRP, 25FT-0009).

PROVENANCE AND PEER REVIEW

Not commissioned; externally peer reviewed. 